No. $14(18)$

\title{
ROUNDING IN THE PROBLEM OF THE ALLOCATION OF INDIVISIBLE GOODS
}

\author{
Katarzyna Cegiełka, Janusz Łyko
}

\begin{abstract}
Using approximate, rounded values implies, in a sense, that an exact numerical value may be ignored. In many cases the difference between the exact and approximate values is not important, and replacing exact numbers by their approximate values does not result in undesired consequences. Yet in certain circumstances, rounding significantly influences the solutions of given problems. This is the case, among others, when we allocate indivisible goods. It may happen that the rounding mode affects the result of allocation so much that the rounding differences cannot be neglected by the agents participating in distribution. This paper presents the classic problem of distributing mandates in representative bodies along with different rounding modes in respective solution procedures.
\end{abstract}

Keywords: rounding rule, approximation, allocation problem, indivisible goods.

JEL Classification: D39

DOI: $10.15611 / \mathrm{dm} .2017 .14 .01$

\section{Introduction}

In practical applications, approximate numbers are often used instead of exact numerical values. The standard example is the number $\pi$. The decisive majority of people asked about the number $\pi$ will answer 3.14, i.e. will use its approximate value, often unconsciously. The advancement of technology supports such thinking. All computers and calculators display approximate values of numbers whose decimal representations exceed the accuracy of those devices. All agree to such a state of affairs and, mostly unconsciously, accept it. Approximate values are very often given when dealing with averages. Average scores of pupils and students, average monthly temperatures, and the average price of a given good in a given market represent instances when giving exact numbers is often impossible or simply unnecessary. Approximations are easily accepted, especially when they do not significantly

\footnotetext{
Katarzyna Cegiełka

Wrocław University of Economics

e-mail: katarzyna.cegielka@ue.wroc.pl

ORCID: 0000-0001-5208-1074
}

\author{
Janusz Lyko \\ Wrocław University of Economics \\ email: janusz.lyko@ue.wroc.pl \\ ORCID: 0000-0002-0332-1921
}


distort the nature of things. It is well-known, for example, that in some engineering applications one may cut off a certain part of a number's fraction without any harm to the usability of the produced machine. Certainly, a different accuracy is needed when producing advanced optical instruments compared with prefabricated building units, but suitable approximations are not challenged.

Similar behaviour can also be detected in the area of economic applications. Prices of goods are very often remembered as approximations. When buying a car in Poland, the buyer is focused on thousands of zloty, ignoring hundreds, tens, units and 'grosze'('grosz' i.e. $100^{\text {th }}$ of a Polish 'złoty'). When repairing a car or buying spare parts, one forgets grosze as a rule, while the price in zlotys is remembered. At a filling station one deals with the exact price of fuel in zlotys and grosze. Such a behaviour notably result from the significance of the ignored parts of the price of a given good or service. The greater the amounts of currency we deal with, the easier the approximations with smaller accuracy.

The prices of goods are often rounded as a consequence of legal regulations. With the introduction of the euro, one common currency, all prices had to be re-denominated, often after suitable rounding. Later on, for example, all prices in Finland have been rounded up to five cents, with one eurocent and two eurocent coins practically eliminated from the circulation. A similar situation also exists in Poland. It is emphasized that the number of one grosz and two grosz coins needed to pay for goods is so large that most people leave them at home in order to carry wallets or purses which weigh less. As a result, those coins disappear from the market and have to be replenished. The follow-on costs of such operations can exceed the gains of pricing by means of more exact values.

The disappearance of tens or even hundreds of units of given currencies is often commonplace in times of heavy inflation. Increasingly the higher prices of goods reduce the purchasing power of smaller units of currency which are then withdrawn from circulation. For example, at present in Iran the smallest unit of currency, the Iranian rial, is a 50 rial coin, and consequently all prices of goods and services are rounded up to this value. The rial is not divided into smaller units, corresponding to cents (or grosze). On a large scale this phenomenon has been recently observed in Zimbabwe, with a 100,000,000,000,000 Zimbabwean dollar banknote circulating there in the market before its subsequent withdrawal and denomination. Due to hyperinflation in this country, neither thousands or millions could express the prices of goods, therefore large numbers had to be used to describe the market value of goods. 
In all the examples above, when approximating, one deals with a quantity which differs from the exact numerical value to a practically insignificant degree. As a consequence, the rule leading to a particular approximation does not have to be specified. The problem of distributing indivisible goods is different because rounding is to integers, while cut-off fractions can create a situation where a given agent will be allocated a unit of the good or will be not. A typical example here is how the representations in collective bodies are determined, and particularly how the mandates are distributed in various legislative bodies endowed with decision making authority, such as councils or parliaments. The role and significance of the rules leading to rounding are so critical that they must be settled via political negotiations. Those rules have also been mathematically analyzed in detail as regards the expected social equity. Considering that approximate values have to be indicated, in many aspects an insignificant problem, the wealth of the proposed solutions is really striking.

\section{Distribution of indivisible goods}

In the realm of European culture, the fundamental rule of distributing gains and burdens was formulated by Aristotle [2002], according to which each agent participating in distribution should be allocated a share of good or burden in proportion to each agent's contribution to the total worth of all agents. The worth is typically given numerically, but its interpretation can be significantly diverse. When a firm's profit is distributed, the worth of an agent is represented by the number of shares held; when the cost of a common investment is divided, it is a degree to which respective investors benefit from it, whereas in the case of determining a social representation under study, it is the population in the respective constituencies. At present, the proportional rule is not challenged as a means to shape parliamentary representations. Nevertheless, debate continues as regards the mode of rounding the results obtained according to the rule, because the total worth of agents, i.e. the total population of all constituencies exceeds by far the number of all mandates, therefore the rounding problem is practically unavoidable.

The problem was first solved [Young 1994] around the beginning of the 19th century, when the seats in the United States House of Representatives were apportioned to the representatives of the states. The so-called divisor methods proposed at that time and their equivalent variants are used in practice in many democracies today. The divisor methods are obviously consistent with the classic Aristotelian principle of proportional allocation. 
Formally, the issue can be represented as follows. Let $p=\left(p_{1}, p_{2}, \ldots, p_{m}\right)$ be a vector of positive natural numbers, with $p_{i}$ denoting the population in constituency $i$, and $H \geq 0$ - the total number of seats to be allocated. The vector of quotas $q=\left(q_{1}, q_{2}, \ldots, q_{m}\right)$ where $q_{i}=\frac{p_{i} H}{\sum_{i=1}^{m} p_{i}}$, determines the allocation that is proportional with respect to populations in individual constituencies. However, in most cases the quantities $q_{i}$ are not natural numbers. Hence, the problem consists in determining a vector of nonnegative natural numbers $a=\left(a_{1}, a_{2}, \ldots, a_{m}\right)$ such that $\sum_{i=1}^{m} a_{i}=H$ [Baliński, Young 1980].

The divisor methods solving the problem of seat allocation in representative bodies are by Jefferson, Adams, Webster, Hill-Huntington and Dean. The first method was put forward by Thomas Jefferson, the first United States Secretary of State. According to his procedure, the divisor $d$ is determined leading to the sequence of modified quotas $q^{\prime}=\left(q_{1}^{\prime}, q_{2}^{\prime}, \ldots, q_{m}^{\prime}\right)$, where $q_{i}^{\prime}=\frac{p_{i}}{d}$. Next, each constituency is allocated $\left\lfloor\frac{p_{i}}{d}\right\rfloor$ mandates. If $\sum_{i=1}^{m}\left\lfloor\frac{p_{i}}{d}\right\rfloor>H$, the divisor $d$ should be increased, if $\sum_{i=1}^{m}\left\lfloor\frac{p_{i}}{d}\right\rfloor<H$ - decreased [Lauwers, Puyenbroeck 2006].

The Adams and Webster methods are simple modifications of the Jefferson method. The author of the first one believed that the quantities $\frac{p_{i}}{d}$ should be rounded upwards, therefore the divisor $d$ should be determined so that $\sum_{i=1}^{m}\left[\frac{p_{i}}{d}\right]=H$ holds. In the Webster method the fractions are rounded to the nearest integer [Baliński, Young 1980].

The Hill-Huntington method and the Dean method are also based on modified quotas, with rounding of their values applied to the geometric mean and harmonic mean, respectively. That is, the ith constituency is allocated $\left\lfloor q_{i}^{\prime}\right\rfloor$ mandates, if its modified quota is smaller than the geometric (harmonic) mean of its two nearest integers, and $\left\lceil q_{i}^{\prime}\right\rceil$ otherwise [Baliński, Young 1977]. 
It is easily seen that both mentioned methods differ basically in threshold $t$, i.e. a number from the interval $[n, n+1]$ whose consequence is whether the specific value of allocation, or rounding of the modified quota $q_{i}{ }^{\prime}$, is the lower or the upper endpoint of this interval. In the first three cases the value $t$ does not move its position in the interval depending on $n$. These are its endpoints and its midpoint. The two remaining cases are different because the placement of geometric and harmonic means depends on $n$. Introducing a movable threshold is justified in practice. Cutting off a fractional part signifies something different to an agent entitled to a large amount of a good, than to another agent whose entitlement is smaller. When rounding numbers 100.25 and 1.25 to the nearest integer, we remove 0.25 unit of the good, but in the first item it means only the subtraction of 0.25 percent of belongings, whereas in the other as much as 25 percent. Therefore the threshold $t$ should be varied depending on the integer part of a given number. It is worth emphasizing that in each of the above-mentioned cases a number from the interval $[n, n+1]$ is rounded either to $n$ or to $n+1$. It turns out that generally this is not always true, therefore the creation of the rules leading to acceptable approximations is even more significant. However, in the case of any distribution of indivisible goods this problem has no solution that would satisfy all the participating agents.

\section{Rounding rules}

The problem of rounding is examined in its generality by Pukelsheim [2014]. The book introduces the so-called rounding functions and rounding rules. The definition of a rounding function shows that the approach generalizes the standard ideas of rounding.

Definition 1. A function $f:[0, \infty) \rightarrow \mathbb{N}^{1}$ is a rounding function if it is increasing and onto.

Basic examples of rounding functions are the floor function $\lfloor x\rfloor$, the ceiling function $\lceil x\rceil$ and the nearest integer function $[x]$, which for $x \geq 0$ are defined as follows:

$$
\begin{aligned}
& \lfloor x\rfloor:=\max \{n \in \mathbb{N} \mid n \leq x\}, \\
& \lceil x\rceil:=\min \{n \in \mathbb{N} \mid n \geq x\},
\end{aligned}
$$

\footnotetext{
${ }^{1} \mathbb{N}:=\{0,1,2,3, \ldots\}$ is assumed in the entire paper.
} 


$$
[x]:=\left\{\begin{array}{l}
\lceil x\rceil \text { for } x-\lfloor x\rfloor \geq 0.5 \\
\lfloor x\rfloor \text { for } x-\lfloor x\rfloor<0.5
\end{array} .\right.
$$

Rounding rules are more flexible variants of rounding functions, which in some cases are too weak and unable to solve a particular allocation problem (see Example 1). Modifications of rounding functions into rounding rules for basic examples of the floor function $\lfloor x\rfloor$, the ceiling function $\lceil x\rceil$ and the nearest integer function $[x]$ are, respectively, for $x \geq 0$, the rule of downward rounding $\| x\rfloor \mid$, the rule of upward rounding $\mid\lceil x\rceil$ and the rule of standard rounding $|[x]|$, defined as follows:

$$
\begin{gathered}
\|x\|:=\left\{\begin{array}{ll}
\{\lfloor x\rfloor\} & \text { for } x \neq 1,2,3, \ldots \\
\{x-1, x\} & \text { for } x=1,2,3, \ldots
\end{array},\right. \\
\|x\|:=\left\{\begin{array}{ll}
\{\lceil x\rceil\} & \text { for } x \neq 1,2,3, \ldots \\
\{x, x+1\} & \text { for } x=1,2,3, \ldots
\end{array},\right. \\
\|[x]:= \begin{cases}\{\lceil x\rceil\} & \text { for } x-\lfloor x\rfloor>0.5 \\
\{\lfloor x\rfloor,\lceil x\rceil\} & \text { for } x-\lfloor x\rfloor=0.5 \\
\{\lfloor x\rfloor\} & \text { for } x-\lfloor x\rfloor<0.5\end{cases}
\end{gathered}
$$

For example, for $x_{1}=5, x_{2}=3.7$, we have $\left.\| x_{1}\right\rfloor=\{4,5\},\left\|x_{1}\right\|=\{5,6\}$, $\left.\| x_{2}\right\rfloor=\{3\}$.

Although definitions of downward, upward and standard rounding rules are quite easy to be written with a formula, it is much more convenient to define general rounding rule using the so-called jump-point sequences, where for a given rounding function $f$, its $n$th jump point is defined by the formula $s(n):=\inf \{x \geq 0 \mid f(x) \geq n\}$.

Definition 2. A jump point sequence $s(0), s(1), s(2), \ldots$ is an unbounded sequence satisfying $s(0)=0 \leq s(1)<s(2)<\ldots$. A jump-point sequence defines a rounding rule $\llbracket \cdot \rrbracket$ by setting, for all $x \geq 0$ and $n \in \mathbb{N}$ : 


$$
\llbracket x \rrbracket:=\left\{\begin{array}{lll}
\{0\} & \text { for } & x=0 \\
\{n\} & \text { for } & x \in(s(n), s(n+1)) . \\
\{n-1, n\} & \text { for } & x=s(n)>0
\end{array}\right.
$$

For the basic rounding rules we have jump-point sequences as follows:

$\begin{array}{llllll}\text { Downward rounding }\|x\|: & 0, & 1, & 2, & 3, & \ldots \\ \text { Upward rounding }\|x\|: & 0, & 0, & 1, & 2, & \ldots \\ \text { Standard rounding } \|[x]:: & 0, & 0.5, & 1.5, & 2.5, & \ldots\end{array}$

Notice that all these jump-point sequences place the $n$th jump point in an interval $[n-1, n]$. However, it is not always necessarily so. Consider ${ }^{2}$ the rounding rule with jump points $s(n)=n^{0.9}$ for $n \geq 1$. Then $s(9)=9^{0.9}=7.2$ and $s(10)=10^{0.9}=7.9$, hence 7.5 is rounded to 9 . Another example is a jumppoint sequence with jump points $s(n)=2^{n}$ for $n \geq 1$. We have then $x \in(0,2)$ rounded to $0, x \in(2,4)$ rounded to 1 , and so on. A convenient property of a jump-point sequence placing its $n$th jump-point in an interval $[n-1, n]$ is characteristic for signpost sequences which are, in a sense, more natural.

Definition 3. A signpost sequence $s(0), s(1), s(2), \ldots$ is characterized by the three properties:

1) $s(0)=0$

2) $s(n) \in[n-1, n]$ for all $n=1,2,3, \ldots$,

3) $s(n)=n-1$ for some $n \geq 1 \Rightarrow \forall n \geq 1 s(n)<n$, $s(n)=n$ for some $n \geq 1 \Rightarrow \forall n \geq 1 s(n)>n-1$.

Obviously every signpost sequence is a jump-point sequence, therefore it defines some rounding rule. Classic divisor methods of apportionment are defined by some signpost sequences. However, note that the procedures described in Section 2 relate to the basic rounding functions which are in practice sufficient tools to determine a desired allocation. Yet it is easy to demonstrate examples of cases with the so-called ties, when rounding rules are indispensable.

Example 1. Let us consider six constituencies with the following populations: 840,000, 700,000, 506,092, 364,207, 284,505, 131,261. Then with $H=17$ and the floor rounding function we have: for divisors $d<140,000$, the

\footnotetext{
${ }^{2}$ The provided examples of rounding rules are used for parliamentary elections in Estonia and Macau, respectively [Pukelsheim 2014].
} 
$\operatorname{sum} \sum_{i=1}^{6}\left\lfloor\frac{p_{i}}{d}\right\rfloor \geq 18$, and for divisors $d>140,000$, the sum $\sum_{i=1}^{6}\left\lfloor\frac{p_{i}}{d}\right\rfloor<16$. But when we apply the downward rounding rule, then for the divisor $d=140,000$ we obtain $q_{1}^{\prime}=6, q_{2}^{\prime}=5, q_{3}^{\prime}=3.6, q_{4}^{\prime}=2.6, q_{5}^{\prime}=2.03, q_{6}^{\prime}=0.9 \quad$ and $\left.\left.\left.\left.\left\|q_{1}^{\prime}\right\|=\{5,6\}, \| q_{2}^{\prime}\right\rfloor=\{4,5\}, \| q_{3}^{\prime}\right\rfloor=\{3\},\left\|q_{4}^{\prime}\right\|=\| q_{5}^{\prime}\right\rfloor=\{2\}, \| q_{6}^{\prime}\right\rfloor=\{0\}$. Therefore the allocations $(6,4,3,2,2,0)$ and $(5,5,3,2,2,0)$ are the sought-after solutions [Pukelsheim 2014].

Classic divisor methods are intuitively understandable and usually do not generate practical difficulties. They can be defined by rounding rules.

Definition 4. For given $H \in \mathbb{N}$ and vector $p=\left(p_{1}, p_{2}, \ldots, p_{m}\right)$ of positive numbers a divisor method $A$ is induced by the rounding rule $\llbracket \cdot \rrbracket$ such that $A(H, p):=\left\{\left(a_{1}, \ldots, a_{m}\right) \in \mathbb{N}^{m} \mid a_{1} \in \llbracket \frac{p_{1}}{d} \rrbracket, \ldots, a_{m} \in \llbracket \frac{p_{m}}{d} \rrbracket\right.$ for some $\left.d>0, \sum_{i=1}^{m} a_{i}=H\right\}$.

Thus classic divisor methods are induced by the respective rounding rules whose definitions by signpost sequences are of the form:

Downward rounding (Jefferson method): $\quad s(n)=n$.

Upward rounding (Adams method): $\quad s(0)=0, s(n)=n-1$ for $n \geq 1$.

Standard rounding (Webster method): $\quad s(0)=0, s(n)=n-0.5$ for $n \geq 1$.

Geometric rounding (Hill-Huntington method): $s(n)=\sqrt{(n-1) n}$.

Harmonic rounding (Dean method):

$$
s(n)=\frac{2}{1 /(n-1)+1 / n} .
$$

\section{Significance of rounding}

In Section 2 we argued that rounding to integers ignores fractions, which may be of different importance for agents as regards the amount of eventually allocated goods. Hence the problem arises as to the strength of this fact affecting the selection of rounding rule, so that the obtained solution may be considered fair from the viewpoint of each agent. In order to explain this issue, randomness of deviations between a mean quota and a mean number of allocated mandates was assumed as the criterion of fairness of the allocation, 
and then a simple experiment was performed to test the difference between these two quantities, depending on the rounding rule.

Table 1 . The results of the experiment with constant rounding thresholds in classic divisor methods

\begin{tabular}{|c|c|c|c|c|c|c|c|c|}
\hline \multicolumn{3}{|c|}{ Downward rounding } & \multicolumn{3}{|c|}{ Upward rounding } & \multicolumn{3}{|c|}{ Standard rounding } \\
\hline$\overline{q_{i}}$ & $\overline{a_{i}}$ & $\overline{q_{i}}-\overline{a_{i}}$ & $\overline{q_{i}}$ & $\overline{a_{i}}$ & $\overline{q_{i}}-\overline{a_{i}}$ & $\overline{q_{i}}$ & $\overline{a_{i}}$ & $\overline{q_{i}}-\overline{a_{i}}$ \\
\hline 3.487 & 3.191 & 0.296 & 3.471 & 3.826 & -0.356 & 3.460 & 3.402 & 0.058 \\
\hline 3.890 & 3.610 & 0.280 & 3.858 & 4.170 & -0.312 & 3.862 & 3.859 & 0.003 \\
\hline 4.278 & 4.012 & 0.266 & 4.286 & 4.553 & -0.267 & 4.267 & 4.273 & -0.006 \\
\hline 4.701 & 4.461 & 0.240 & 4.701 & 4.930 & -0.229 & 4.676 & 4.687 & -0.011 \\
\hline 5.090 & 4.880 & 0.210 & 5.115 & 5.337 & -0.222 & 5.090 & 5.090 & 0.000 \\
\hline 5.506 & 5.317 & 0.189 & 5.532 & 5.740 & -0.208 & 5.497 & 5.498 & -0.001 \\
\hline 5.915 & 5.746 & 0.169 & 5.933 & 6.096 & -0.163 & 5.928 & 5.944 & -0.016 \\
\hline 6.334 & 6.179 & 0.155 & 6.364 & 6.508 & -0.144 & 6.353 & 6.353 & 0.000 \\
\hline 6.761 & 6.631 & 0.130 & 6.765 & 6.874 & -0.110 & 6.757 & 6.772 & -0.015 \\
\hline 7.188 & 7.076 & 0.112 & 7.189 & 7.308 & -0.119 & 7.167 & 7.165 & 0.002 \\
\hline 7.606 & 7.525 & 0.081 & 7.594 & 7.671 & -0.077 & 7.593 & 7.567 & 0.026 \\
\hline 8.015 & 7.943 & 0.072 & 8.005 & 8.062 & -0.057 & 7.992 & 8.002 & -0.011 \\
\hline 8.448 & 8.417 & 0.031 & 8.420 & 8.456 & -0.037 & 8.409 & 8.406 & 0.003 \\
\hline 8.852 & 8.831 & 0.021 & 8.833 & 8.826 & 0.007 & 8.843 & 8.852 & -0.010 \\
\hline 9.272 & 9.280 & -0.008 & 9.253 & 9.251 & 0.002 & 9.281 & 9.260 & 0.021 \\
\hline 9.700 & 9.743 & -0.043 & 9.675 & 9.653 & 0.022 & 9.713 & 9.717 & -0.004 \\
\hline 10.109 & 10.149 & -0.040 & 10.091 & 10.030 & 0.061 & 10.160 & 10.158 & 0.002 \\
\hline 10.545 & 10.622 & -0.077 & 10.522 & 10.435 & 0.087 & 10.560 & 10.568 & -0.008 \\
\hline 10.964 & 11.061 & -0.097 & 10.959 & 10.860 & 0.099 & 10.970 & 10.987 & -0.017 \\
\hline 11.391 & 11.525 & -0.135 & 11.371 & 11.238 & 0.133 & 11.413 & 11.392 & 0.021 \\
\hline 11.812 & 11.963 & -0.151 & 11.826 & 11.679 & 0.147 & 11.836 & 11.836 & 0.000 \\
\hline 12.238 & 12.412 & -0.174 & 12.233 & 12.049 & 0.184 & 12.253 & 12.250 & 0.003 \\
\hline 12.664 & 12.848 & -0.184 & 12.680 & 12.479 & 0.201 & 12.685 & 12.692 & -0.007 \\
\hline 13.069 & 13.285 & -0.216 & 13.109 & 12.880 & 0.229 & 13.095 & 13.121 & -0.026 \\
\hline 13.519 & 13.779 & -0.261 & 13.533 & 13.289 & 0.244 & 13.520 & 13.523 & -0.003 \\
\hline 13.957 & 14.218 & -0.261 & 13.968 & 13.694 & 0.274 & 13.938 & 13.940 & -0.002 \\
\hline 14.383 & 14.663 & -0.280 & 14.390 & 14.091 & 0.299 & 14.381 & 14.388 & -0.007 \\
\hline 14.806 & 15.136 & -0.330 & 14.826 & 14.512 & 0.314 & 14.802 & 14.800 & 0.002 \\
\hline
\end{tabular}

Source: own elaboration. 
The experiment was performed as follows. Given the number of agents $m=28$ and the rounding rule, 1000 sequences of populations were randomly chosen, where the population of the ith constituency $p_{i} \in[100,000 ; 500,000]$ and the total number of mandates $H \in[250 ; 259]$. Based on this input, 1000 sequences of quotas and 1000 integer allocations were generated, and respective means were calculated, i.e. mean quotas $\bar{q}_{i}$ and mean numbers of mandates $\overline{a_{i}}$. The results of the experiment are presented in Tables 1 to 3 .

Table 2. The results of the experiment with variable rounding thresholds in classic divisor methods

\begin{tabular}{|c|c|c|c|c|c|}
\hline \multicolumn{3}{|c|}{ Geometric rounding } & \multicolumn{3}{|c|}{ Harmonic rounding } \\
\hline$q_{i}$ & $a_{i}$ & $\bar{q}_{i}-\bar{a}_{i}$ & $\bar{q}_{i}$ & $a_{i}$ & $q_{i}-a_{i}$ \\
\hline 3.460 & 3.439 & 0.021 & 3.460 & 3.470 & -0.010 \\
\hline 3.862 & 3.884 & -0.022 & 3.862 & 3.916 & -0.054 \\
\hline 4.267 & 4.303 & -0.036 & 4.267 & 4.327 & -0.060 \\
\hline 4.676 & 4.706 & -0.030 & 4.676 & 4.719 & -0.043 \\
\hline 5.090 & 5.104 & -0.014 & 5.090 & 5.117 & -0.027 \\
\hline 5.497 & 5.506 & -0.009 & 5.497 & 5.513 & -0.016 \\
\hline 5.928 & 5.955 & -0.027 & 5.928 & 5.964 & -0.036 \\
\hline 6.353 & 6.370 & -0.017 & 6.353 & 6.378 & -0.025 \\
\hline 6.757 & 6.782 & -0.025 & 6.757 & 6.791 & -0.034 \\
\hline 7.167 & 7.170 & -0.003 & 7.167 & 7.176 & -0.009 \\
\hline 7.593 & 7.573 & 0.020 & 7.593 & 7.583 & 0.010 \\
\hline 7.992 & 8.002 & -0.011 & 7.992 & 7.998 & -0.006 \\
\hline 8.409 & 8.403 & 0.006 & 8.409 & 8.404 & 0.005 \\
\hline 8.843 & 8.847 & -0.005 & 8.843 & 8.846 & -0.004 \\
\hline 9.281 & 9.258 & 0.023 & 9.281 & 9.256 & 0.025 \\
\hline 9.713 & 9.710 & 0.003 & 9.713 & 9.704 & 0.009 \\
\hline 10.160 & 10.152 & 0.008 & 10.160 & 10.146 & 0.014 \\
\hline 10.560 & 10.558 & 0.002 & 10.560 & 10.547 & 0.013 \\
\hline 10.970 & 10.974 & -0.004 & 10.970 & 10.955 & 0.015 \\
\hline 11.413 & 11.389 & 0.024 & 11.413 & 11.378 & 0.034 \\
\hline 11.836 & 11.828 & 0.008 & 11.836 & 11.819 & 0.017 \\
\hline 12.253 & 12.237 & 0.016 & 12.253 & 12.227 & 0.026 \\
\hline 12.685 & 12.674 & 0.011 & 12.685 & 12.662 & 0.023 \\
\hline 13.095 & 13.100 & -0.005 & 13.095 & 13.078 & 0.017 \\
\hline 13.520 & 13.498 & 0.022 & 13.520 & 13.483 & 0.037 \\
\hline 13.938 & 13.915 & 0.023 & 13.938 & 13.903 & 0.035 \\
\hline 14.381 & 14.376 & 0.005 & 14.381 & 14.362 & 0.019 \\
\hline 14.802 & 14.783 & 0.019 & 14.802 & 14.772 & 0.030 \\
\hline
\end{tabular}

Source: own elaboration. 
Table 3. The results of the experiment with variable rounding thresholds in chosen signpost sequences

\begin{tabular}{|c|c|c|c|c|c|c|c|c|}
\hline \multicolumn{3}{|c|}{$s_{1}(n)$} & \multicolumn{3}{|c|}{$s_{2}(n)$} & \multicolumn{3}{|c|}{$S_{3}(n)$} \\
\hline$q_{i}$ & $a_{i}$ & $q_{i}-a_{i}$ & $q_{i}$ & $a_{i}$ & $q_{i}-a_{i}$ & $q_{i}$ & $a_{i}$ & $q_{i}-a_{i}$ \\
\hline 3.455 & 3.318 & 0.137 & 3.460 & 3.980 & -0.520 & 3.460 & 3.807 & -0.347 \\
\hline 3.855 & 3.744 & 0.111 & 3.862 & 4.302 & -0.440 & 3.862 & 4.182 & -0.320 \\
\hline 4.251 & 4.141 & 0.110 & 4.267 & 4.670 & -0.403 & 4.267 & 4.526 & -0.259 \\
\hline 4.667 & 4.575 & 0.092 & 4.676 & 5.038 & -0.362 & 4.676 & 4.920 & -0.244 \\
\hline 5.081 & 4.968 & 0.113 & 5.090 & 5.409 & -0.319 & 5.090 & 5.299 & -0.209 \\
\hline 5.493 & 5.403 & 0.090 & 5.497 & 5.796 & -0.299 & 5.497 & 5.701 & -0.204 \\
\hline 5.927 & 5.831 & 0.096 & 5.928 & 6.186 & -0.258 & 5.928 & 6.104 & -0.176 \\
\hline 6.353 & 6.267 & 0.086 & 6.353 & 6.579 & -0.226 & 6.353 & 6.500 & -0.147 \\
\hline 6.754 & 6.695 & 0.059 & 6.757 & 6.942 & -0.185 & 6.757 & 6.889 & -0.132 \\
\hline 7.158 & 7.096 & 0.062 & 7.167 & 7.303 & -0.136 & 7.167 & 7.246 & -0.079 \\
\hline 7.587 & 7.512 & 0.075 & 7.593 & 7.714 & -0.121 & 7.593 & 7.661 & -0.068 \\
\hline 7.986 & 7.956 & 0.030 & 7.992 & 8.082 & -0.091 & 7.992 & 8.055 & -0.063 \\
\hline 8.403 & 8.376 & 0.027 & 8.409 & 8.470 & -0.061 & 8.409 & 8.451 & -0.042 \\
\hline 8.840 & 8.818 & 0.021 & 8.843 & 8.861 & -0.019 & 8.843 & 8.862 & -0.020 \\
\hline 9.282 & 9.261 & 0.021 & 9.281 & 9.237 & 0.044 & 9.281 & 9.258 & 0.023 \\
\hline 9.719 & 9.709 & 0.010 & 9.713 & 9.659 & 0.054 & 9.713 & 9.671 & 0.042 \\
\hline 10.163 & 10.164 & -0.001 & 10.160 & 10.071 & 0.089 & 10.160 & 10.097 & 0.063 \\
\hline 10.568 & 10.591 & -0.023 & 10.560 & 10.438 & 0.122 & 10.560 & 10.467 & 0.093 \\
\hline 10.981 & 11.028 & -0.047 & 10.970 & 10.820 & 0.150 & 10.970 & 10.873 & 0.097 \\
\hline 11.420 & 11.464 & -0.044 & 11.413 & 11.227 & 0.185 & 11.413 & 11.295 & 0.118 \\
\hline 11.840 & 11.908 & -0.068 & 11.836 & 11.595 & 0.241 & 11.836 & 11.686 & 0.150 \\
\hline 12.251 & 12.329 & -0.079 & 12.253 & 12.005 & 0.248 & 12.253 & 12.082 & 0.171 \\
\hline 12.685 & 12.807 & -0.122 & 12.685 & 12.380 & 0.305 & 12.685 & 12.477 & 0.208 \\
\hline 13.102 & 13.234 & -0.132 & 13.095 & 12.757 & 0.338 & 13.095 & 12.877 & 0.218 \\
\hline 13.526 & 13.653 & -0.127 & 13.520 & 13.151 & 0.369 & 13.520 & 13.286 & 0.234 \\
\hline 13.951 & 14.104 & -0.153 & 13.938 & 13.536 & 0.402 & 13.938 & 13.656 & 0.282 \\
\hline 14.395 & 14.547 & -0.152 & 14.381 & 13.954 & 0.427 & 14.381 & 14.081 & 0.300 \\
\hline 14.810 & 15.004 & -0.194 & 14.802 & 14.338 & 0.464 & 14.802 & 14.485 & 0.317 \\
\hline
\end{tabular}

Source: own elaboration. 
Tables 1 and 2 include the results of the experiment for classic divisor methods. Table 1 presents the results for the Jefferson, Adams and Webster methods, i.e. for the constant rounding thresholds. Table 2 presents the results for the Hill-Huntington and Dean methods, the classic methods with variable rounding threshold, i.e. depending on the endpoints of the interval $[n, n+1]$. Table 3 in turn presents the results for the variable rounding thresholds, chosen by the authors, and defined by the signpost sequences of the form $s_{1}(n)=n /(n+1), s_{n}(n)=n / m$ and $s_{3}(n)=n / \sum_{i=1}^{m} p_{i}$.

\section{Conclusions}

Based on the performed experiment, one may argue that considering the difference between the mean quota and the mean number of allocated mandates, the only neutral rounding method which does not favour any constituency is rounding to the nearest integer. This result is consistent with the expectations implied by the theorem [Young 1994], stating that the only constant threshold, neutral in this sense, is the centre of the segment. Upward or downward rounding methods, when the threshold $t$ is the lower or the upper endpoint of the interval, evidently favours small or great constituencies, respectively. The thresholds determined by geometric and harmonic means also favour smaller constituencies, although to a smaller degree. This is a consequence of the fact that both means are smaller than the arithmetic mean. It is also known that their convergence to the arithmetic mean is very quick, as $n$ increases. As soon as with $n=25$, the difference between the arithmetic mean and the harmonic mean of $n$ and $n+1$ is smaller than 0.01. In the case of the geometric mean such a difference is achieved even sooner, for $n=14$. Therefore, when assuming the geometric mean as the rounding threshold, one may pretend to safeguard the interests of the smaller constituencies on one hand, while on the other to move towards an unbiased solution. This property of the Hill-Huntington method may have contributed to its great popularity in the U.S. and it has been used as the apportionment method of the seats in the United States House of Representatives among the states since 1941, when it was endorsed by law.

The application of variable thresholds introduces only minor changes. Even if they recognize different weights of fractional parts depending on the amount of goods held by agents, but still the trap of favouring smaller or greater electoral districts is unavoidable. The preferences move actually towards either direction depending on the position of the threshold against the 
centre of the interval $[n, n+1]$. That is why the signpost sequences considered in our experiment: $s_{1}(n)=n /(n+1), s_{n}(n)=n / m$ and $s_{3}(n)=n / \sum_{i=1}^{m} p_{i}$, behave similarly to constant thresholds as regards favouring smaller or greater agents: the first one behaves like a signpost sequence defining downward rounding, and the two remaining like a signpost sequence defining upward rounding.

Therefore one can plainly see that the choice of rounding rule cannot comply with the distributive justice, even if it is reduced only to the two factors - recognizing a heavier weight of cancelled parts in the case of smaller constituencies, and matching the distribution with the actual proportions represented by the modified quotas. Needless to say, this problem becomes irrelevant when all electoral districts have approximately equal populations. However it becomes increasingly aggravated as differences widen, and then it can provoke giving a preference to smaller agents. As a result, current electoral systems include diverse solutions, for example the Webster method is applied by electoral systems in New Zealand, Norway and Sweden, implying that legislators assign more weight to the compliance of allocation with a modified quota and do not allow the biased treatment of any electoral districts. The legally binding solution in the U.S. at present, with geometric mean rounding, can be seen as the wish to find a halfway solution. Finally, the distribution of seats in the European Parliament, known as the Cambridge Compromise, seems to be an encouragement given to the least populous member countries in the European Union, because it ensures a degressively proportional allocation imposed by the Treaty of Lisbon, as well as including a proportional component with upward rounding to the nearest integer. This solution is not a surprise though if we compare the populations of Malta and Germany - the least and the most populous state in the community.

\section{Bibliography}

Aristotle (2002). Nicomachean Ethics - Book 5. Selected Works. Wydawnictwo Naukowe PWN. Warszawa.

Baliński M., Young H.P. (1977). On Huntington methods of apportionment. Journal on Applied Mathematics. No 33 (4). Pp. 607-618.

Baliński M., Young H.P. (1980). The Webster method of apportionment. Proceedings of National Academy of Sciences. No 77. Pp. 1-4. 
Lauwers L., Puyenbroeck T.V. (2006). The Hamilton apportionment method is between the Adams and the Jefferson method. Mathematics of Operations Research. No 31 (2). Pp. 390-397.

Pukelsheim F. (2014). Proportional Representation. Apportionment Methods and Their Applications. Springer.

Young H.P. (1994). Equity: In Theory and Practice. Princeton U. Press. Princeton. 